\title{
Construction of a Linkage Map and Identification of Resistance Gene Analog Markers for Root-knot Nematodes in Wild Peach, Prunus kansuensis
}

\author{
Ke Cao, Lirong Wang', Gengrui Zhu, Weichao Fang, Chenwen Chen, and Pei Zhao \\ Zhengzhou Fruit Research Institute, Chinese Academy of Agriculture Sciences, Zhengzhou, China
}

\begin{abstract}
Additional INDEX words. genetic linkage map, resistance gene analog, root-knot nematode, peach
Abstract. The root-knot nematode (RKN) Meloidogyne incognita can cause severe crop loss in economically important Prunus species like peach $(P$. persica), almond $(P$. communis), plum $(P$. salicina), and apricot $(P$. armeniaca). Some peach rootstock, including Nemaguard $(P$. persica), Nemared $(P$. persica), and Myrobalan plum $(P$. cerasifera), display significant resistance to RKN. We present a genetic linkage map constructed by using simple sequence repeat (SSR) and sequence-related amplified polymorphism (SRAP) for a peach backcross population (190 individuals) of RKNresistant 'Honggengansutao' $(P$. kansuensis) and susceptible 'Bailey' $(P$. persica $)$. Degenerate primers designed from conserved motifs of known plant resistance gene $(R)$ products were used to amplify genomic DNA sequences. Twentytwo resistance gene analog (RGA) sequences were selected from 48 RGAs with open-reading frames to design sequence-tagged site markers. The linkage map of 'Honggengansutao' is composed of 138 loci (30 SSRs, 102 SRAPs, five RGAs, and one morphological marker for RKN resistance) assigned to eight linkage groups. The map covers 616 $\mathrm{cM}$ of the peach genome with an average marker spacing of $4.9 \mathrm{cM}$. The five RGAs were mapped to Groups 2,7 , and 8 . One gene (designated $P$ kMi) involved in resistance to RKN was mapped to Group 2 (which also includes the known RKN-resistance RMia gene). BLASTN analysis mapped all RGAs to the peach genome sequence. The map constructed in the study will aid future rootstock breeding with marker-assisted selection to identify additional candidate RGA sequences.
\end{abstract}

Root-knot nematodes are damaging pests of fruit tree crops and numerous other perennial or annual plants. Several rootstock breeding programs using interspecific hybridization have introduced useful traits for size control, adaptation to the new environments, and pest resistance (Desmond and Daniele, 2008). Three major RKN species, Meloidogyne incognita (MI), M. javanica (MJ), and $M$. arenaria (MA), are present in most areas with tropical and Mediterranean climates (Lamberti, 1979; Sasser, 1977). Different levels of RKN resistance have been observed in Prunus species and the corresponding resistance $(R)$ genes used for rootstock breeding (Esmenjaud et al., 1997). The Myrobalan plum clone P.2175 is highly resistant to all tested RKN species, including MI, MA, MJ, and M. floridensis (Lecouls et al., 1997; Rubio-Cabetas et al., 1999). Resistance is conferred by the major dominant gene $M a$. The $M a$ gene, carried by myrobalan plum and almond, has been mapped to linkage Group 7 (G7) of the Prunus reference map (Claverie et al., 2004; Dirlewanger et al., 2004; Van Ghelder et al., 2010). The RMia gene also is a dominant gene carried by the peach cultivars Nemaguard and Nemared (Ramming and Tanner, 1983) and the almond $\times$ peach hybrid 'GF.557' (Claverie et al., 2004) confers resistance limited to MI and MA. The RMia gene maps to linkage Group 2 (G2) of both the Prunus reference map (Dirlewanger et al., 2004) and the 'P.2175' $\times$ 'Felinem' map (Claverie et al., 2004)

The use of degenerate $R$ gene primers has proven very effective for identifying $R$ genes from diverse species of plants (Aarts et al., 1998; Robert et al., 2008; Wang et al., 2010). Crop

Received for publication 30 Sept. 2010. Accepted for publication 10 Feb. 2011. This project was cosupported by the National Natural Science Foundation of China (NSFC) (No. 30771480) and by the National Hi-Tech Research and Developement Program of China (2006AA100108-4-12).

${ }^{1}$ Corresponding author. E-mail: wlirong2009@sina.com. scientists have benefited greatly from the identification and mapping of $R$ genes linked to pathogen and pest resistance phenotypes. Over the last 15 years, over $40 R$ genes, conferring resistance to several pathogens, have been characterized in both model plants and crop species (Qiang et al., 2005). The most abundant class of $R$ genes encodes proteins containing nucleotide-binding sites and leucine-rich repeat (NBS-LRR) domains (Dangl and Jones, 2001). Many NBS-LRR homologs, also known as RGAs, are phylogenetically related to known $R$ genes, and a number of studies have found homologs mapping to $R$ gene loci (Hinchliffe et al., 2005; Gaspero et al., 2007) providing further evidence that such genomic regions code for resistance.

In this report, we have characterized an $R$ gene $(P k M i)$ from $P$. kansuensis 'Honggengansutao'. A genetic linkage map was constructed by using SSR, SRAP, and RGA-sequence-tagged site (STS) markers for mapping RKN resistance. This map is a valuable tool for locating the regions involved in RKN resistance and for the development of additional molecular markers within these regions.

\section{Materials and Methods}

Plant material. Seven $P$. persica rootstock cultivars and two $P$. kansuensis cultivars were used in this study. Resistance to RKN was evaluated in 1998 at Zhengzhou Fruit Institute, Zhengzhou, China. In 2000, 'Honggengansutao' (immune to $M$. incognita) was crossed as a female parent to 'Bailey' (susceptible to $M$. incognita) to obtain $\mathrm{F}_{1}$ hybrids. A randomly selected $\mathrm{F}_{1}$ hybrid was then crossed with 'Bailey' to generate a $\mathrm{BC}_{1}$ population expected to segregate for RKN resistance. Seeds from $\mathrm{BC}_{1}$ were stratified and the subsequent seedlings were planted in a greenhouse in 2005. One hundred ninety individuals were used to construct the genetic map. 
RoOT-KNOT NEMATODE ISOLATES AND EVALUATION OF PLANT MATERIAL. A single MI population was used to evaluate resistance to RKN. The MIs were isolates reared from a single egg mass and maintained on tomato (Solanum lycopersicum). The identity of the population, at the species level, was verified each year before inoculation by means of their morphological characteristics. For example, the female stylet of the MI population is unique; the anterior half of the stylet cone is cylindrical and distinctly curved dorsally. The shaft is only slightly wider posteriorly. The stylet knobs of MI are set off from the shaft.

All plant materials (parents, controls, $\mathrm{F}_{1}$, and $\mathrm{BC}_{1}$ ) were grown in 10-L containers with sterile soil in an open field. Thirty seedlings of nine rootstock cultivars represented their parents, and only one seedling of $\mathrm{BC}_{1}$ hybrids represented itself. When the seedling stems were $\approx 5 \mathrm{~mm}$ in diameter, they were inoculated individually with 500 s-stage MI juveniles (J2s) in May. Four to 5 months after inoculation, the seedlings were washed and roots rated for gall severity as previously described (Barker, 1985) with $0=$ no gall, $1=1 \%$ to $10 \%$ of root system galled, $2=11 \%$ to $30 \%, 3=31 \%$ to $70 \%, 4=71 \%$ to $90 \%$, and $5=$ greater than $90 \%$ galled. The $\mathrm{F}_{1}$ populations were evaluated once, whereas the $\mathrm{BC} 1$ evaluations were repeated for 3 years (consecutive). Each evaluation of RKN resistance included population parents and control plants (tomato).

DNA Extraction. Genomic DNA was isolated from fresh leaf tissue using a DNA extraction kit (Tiangen, Beijing, China) according to the manufacturer's instructions. The DNA concentrations were checked on 1\% TAE agarose gels and working solutions of genomic DNA (100 ng. $\left.\mu \mathrm{L}^{-1}\right)$ were kept in TE buffer ( $\mathrm{pH}$ 8.0).

Simple SEQUENCE REPEAT ANALYSIS. For SSR markers, the optimal annealing of 134 primer pairs was determined using Temperature Gradient PCR (Biometra, Goettingen, Germany). The polymerase chain reaction (PCR) products were run on $1.5 \%$ agarose gels to verify amplification and to determine optimal annealing temperatures. Successfully amplified SSRs were screened in both parents and a set of eight mapping progeny chosen to establish segregation patterns. Segregating SSRs were then studied in the whole mapping population. The PCR reaction mixture $(10 \mu \mathrm{L})$ contained $0.6 \mu \mathrm{L}$ dNTP $(2.5$ $\mathrm{mM}), 1 \mu \mathrm{L} 10 \times$ PCR buffer (with $\mathrm{Mg}^{2+}$ ), $0.35 \mu \mathrm{L}(10 \mathrm{~mm})$ forward and reverse primers, $10 \mathrm{ng}$ of genomic DNA, 0.5 U Taq DNA polymerase (Promega, Beijing, China), and $6.6 \mu \mathrm{L}$ double-distilled water. PCR reactions were performed in a Biometra T1 thermal cycler under the following conditions: an initial denaturation for $3.5 \mathrm{~min}$ at $94{ }^{\circ} \mathrm{C}$ followed by 30 cycles of $45 \mathrm{~s}$ at $94{ }^{\circ} \mathrm{C}$, then $45 \mathrm{~s}$ at 49 to $57^{\circ} \mathrm{C}$, then $1 \mathrm{~min}$ at $72^{\circ} \mathrm{C}$, and a final extension of $5 \mathrm{~min}$ at $72{ }^{\circ} \mathrm{C}$. The PCR products were visualized by electrophoresis on $8 \%$ polyacrylamide gels run for $1 \mathrm{~h}$ at $250 \mathrm{~W}$ with $1 \times \mathrm{TBE}$.

SEQUENCE-RELATED AMPLIFIED POLYMORPHISM ANALYSIS. For SRAP analysis, 14 forward primers (me1 to me14) and 17 reverse primers (em1 to em17) were used in 238 primer combinations. Amplification was performed as described (Li and Quiros, 2001) and the PCR products were visualized using $1.5 \%$ agarose gels.

Degenerate PRIMER DESIGN AND POLYMERASE CHAIN REACTION CONDITIONS FOR THE RESISTANCE GENE ANALOG. A pair of degenerate primers was designed based on the following NBS-LRR encoding $R$ genes $N$ (GenBank AB120513) and $M$ (GenBank U73916) from the TIR-NBS-LRR class and RPS2 (GenBank NM_118742) and RPM1 (GenBank NM_111584) from the non-TIR-NBS-LRR class. The PCR reaction mixture and thermocycle protocols were the same as those used for SSR analysis.

Amplified products of degenerate RGA primer pairs were separated on $1.2 \%$ agarose gels in $1 \times$ TAE buffer. Desired bands were purified using a Tiangel Midi PCR purification kit (Tiangen), cloned into the pGEM-T easy vector (Promega), and then transferred into Escherichia coli competent cells (DH5 $\alpha$ ). Sequencing of the DNA was performed using an ABI 3730 automated sequencer (BGI, Shenzhen, China). The DNA sequences obtained from 'Honggengansutao' were processed to remove vector sequences using the National Center of Biotechnology Information (NCBI) VecScreen program (NCBI, 2010a). The amino acid sequences of RGAs were deduced on the basis of sequence similarity using the software DNAman (Lynnon Corp., Pointe-Claire, Quebec, Canada). To convert the RGAs with uninterrupted open-reading frame (ORF) sequences into RGA-STS markers, gene-specific primers were designed by primer-blast software (NCBI, 2010b). The RGA-STS primers developed from the sequences of the RGAs produced polymorphic fragments in the $\mathrm{BC}_{1}$ population. The specific PCR reactions with the RGA-STS primers were also performed in $10 \mu \mathrm{L}$ and used the same program in RGA cloning. A cleaved amplified polymorphic (CAP) marker was also created by digesting the RGA-STS products with EcorI, MseI, Sau3AI, AluI, or HaeIII (Takara, Dalian, China). The whole $\mathrm{BC}_{1}$ population was then amplified and digested by the optimum enzyme in $8 \mu \mathrm{L} \mathrm{H}_{2} \mathrm{O}, 1 \mu \mathrm{L} 10 \times$ buffer tango (Fermentas, Shenzhen, China), and $0.25 \mu \mathrm{L}$ enzyme $(2.5 \mathrm{U})$ at $37^{\circ} \mathrm{C}$ for $3 \mathrm{~h}$.

SCORING OF MARKERs. There were four classes of markers scored in SSR, SRAP, and RGA-STS statistics: 1) heterozygous markers segregating for the female parent; 2) heterozygous markers segregating for the male parent; 3) dominant markers where the band was contributed by the female 'Honggengansutao' but was absent in the male parent 'Bailey'; and 4) dominant markers where the band was contributed by the male parent, 'Bailey', but was absent in the female parent, 'Honggengansutao'. The RKN resistance of peach is controlled by a dominant gene originating from 'Honggengansutao', so only markers (1) and (3) that segregated in a 1 present: 1 absent ratio were included in the mapping files. Chi-square tests of goodness of fit were performed on segregation data for all markers to determine their agreement with the expected ratios.

LinkAge MAP CONSTRUCTION. A female parent genetic linkage map for the $\mathrm{BC}_{1}$ family was constructed using JoinMap 2.0 software (Stam and Van Ooijen, 1995). The mapping analysis was conducted using a minimum logarithm of odds threshold (LOD) score of three and a 0.40 maximum recombination frequency. The map distances were computed using the Kosambi mapping function.

Physical distribution of resistance gene analogs on Chromosomes of 'Honggengansutao' PeACH. Twenty-two RGAs were mapped by linkage analysis. BLASTN analysis of RGA sequences against the $\mathrm{Pp}$ whole genome sequence (Version 1.0; Washington State University, 2010a) was used to infer their location on specific chromosomes. The RGAs with same alignment results were thought as a "false" RGA.

\section{Results}

ROOT-KNOT NEMATODE RESISTANCE TESTS OF THE CULTIVARS AND CHOICE OF THE PARENTS FOR $\mathbf{F}_{1}$ AND BC 1 CROSSES. In the nine 
cultivars tested, the high inoculum pressure allowed separation into immune (gall index $=0)$, resistant $(1.0>$ gall index $>0)$, and susceptible (gall index $>1.0$ ) classes (Table 1). The peaches 'Bailey', 'Shouxingtao 2', and 'Shouxingtao 3' were susceptible to RKN, whereas 'Maotao 1', 'Maotao 2', and 'Maotao 3' were resistant. 'Shouxingtao 1', 'Honggengansutao', and 'Baigengansutao' were immune. Thus, 'Bailey' and 'Honggengansutao' were selected as parents for creation of $\mathrm{BC}_{1}$ population.

Resistance SegRegation analysis OF THE BC $_{1}$ POPUlation. One hundred ninety individuals from the ('Honggengansutao' $\times$ 'Bailey') $\times$ 'Bailey' population were screened for RKN resistance. Plants were classified as resistant or susceptible based on the gall index. Eighty-seven individuals were resistant and 103 susceptible. Resistant individuals were coded as heterozygous for the trait and those susceptible were coded as homozygous recessives. The segregation ratio $87 \mathrm{R}: 103 \mathrm{~S}$ was close to $1 \mathrm{R}: 1 \mathrm{~S}$ expected from the $P k M i$ (chi-square $=1.35$; threshold value $=3.84$ for $P=0.95$ ).

Simple Sequence Repeat anAlysis. The 134 SSRs tested for their polymorphisms originated from several Prunus species: 102 were from peach, 21 from japanese plum ( $P$. salicina), six from almond, four from sweet cherry ( $P$. avium), and one from sour cherry ( $P$. cerasus) (Table 2$)$. Differences in the rate of polymorphism were observed among the different sets of SSRs. The UDP set gave the highest rate of polymorphism (60.0\%), whereas the BPPCT, CPPCT, and CPDCT

Table 1. Evaluation of root-knot nematode resistance in nine Prunus rootstocks.

\begin{tabular}{|c|c|c|c|}
\hline Cultivar & Origin & $\begin{array}{l}\text { Gall index } \\
(0-5 \text { scale })^{z}\end{array}$ & Evaluation $^{\mathrm{y}}$ \\
\hline$\overline{\text { Maotao } 1(P . \text { persica })}$ & Southwestern China & 0.71 & Resistant \\
\hline Maotao $2(P$. persica $)$ & Henan, China & 0.18 & Resistant \\
\hline Maotao 3 (P. persica) & United States & 0.36 & Resistant \\
\hline Bailey $(P$. persica $)$ & Canada & 4.11 & Susceptible \\
\hline Shouxingtao 1 ( $P$. persica) & Shanghai, China & 0.00 & Immune \\
\hline Shouxingtao 2 ( $P$. persica) & Shanghai, China & 3.75 & Susceptible \\
\hline Shouxingtao $3(P$. persica $)$ & Shanghai, China & 4.46 & Susceptible \\
\hline Honggengansutao (P. kansuensis) & Gansu, China & 0.00 & Immune \\
\hline Baigengansutao ( $P$. kansuensis) & Henan, China & 0.00 & Immune \\
\hline
\end{tabular}

${ }^{\mathrm{z}} 0=$ no gall, $1=1 \%$ to $10 \%$ of root system galled, $2=11 \%$ to $30 \%, 3=31 \%$ to $70 \%, 4=71 \%$ to $90 \%$, and 5 greater than $90 \%$ galled; data are means of 30 replicates.

${ }^{\mathrm{y}}$ Immune (gall index $\left.=0\right)$, resistant $(1.0>$ gall index $>0$ ), and susceptible (gall index $>1.0$ ). sets had rates of $27.0 \%, 37.5 \%$, and $33.3 \%$, respectively (Table 2).

Among the SSRs producing an amplification product, 20 had complex profiles on polyacrylamide gels, 71 revealed no polymorphisms, 30 revealed polymorphisms in 'Honggengansutao', five revealed polymorphisms in 'Bailey', and eight were polymorphic in both parents. Because the $R$ gene originated from 'Honggengansutao', SSRs with easily readable polymorphism profiles in 'Honggengansutao' and in both parents that had an amplification product were used.

SEQUENCE-RELATED AMPLIFIED POLYMORPHISM ANALYSIS. Of 238 SRAP primer pairs tested, 53 primer pair combinations generated polymorphic bands that revealed 638 polymorphic loci (mean of 12.0 polymorphic bands per primer pair; range, 1 to 15 ). Each primer pair produced 10 to 20 clear bands $\approx 100$ to $1000 \mathrm{bp}$ in length. Two hundred eighty-six of the SRAP fragments in the $\mathrm{BC}_{1}$ hybrid and 115 in the 'Honggengansutao' segregated in a 1:1 ratio.

Cloning of NBS-Type Resistance gene analogs and RESISTANCE GENE ANALOG-SEQUENCE-TAGGED SITE ANALYSIS. The forward primer (5'-GGIGGIRTIGGIAAIACIAC-3') was designed on the basis of the NBS motif [GG(L/V/S/I) GKTT] in the sense direction, and the reverse primer (5'-AGIGYIA GIGGIAGICC-3') was based on the membrane-spanning motif (GLPLAL) in the antisense direction. Amplification by PCR using a pair of degenerate primers resulted in a single DNA gel band of $\approx 550 \mathrm{bp}$ that was recovered and cloned. A total of 300 clones was generated from insert-containing recombinant plasmids, and 100 positive clones were retained for further sequencing analysis. After removing the vector and the repetitive sequences, 75 different sequences, NBS1 to NBS75, were identified. Forty-five (60\%) sequences showed significant similarity to known Arabidopsis thaliana $R$ genes and homologs (E-value $10^{-5}$ or less) based on searches using the BLASTX program.

Forty-eight of 75 RGAs with uninterrupted ORFs were deposited in GenBank (HM219232-HM219254,

Table 2. Origin of the simple sequence repeat (SSR) markers used for linkage map construction in the seedling of the Prunus kansuensis 'Honggengansutao' $\mathrm{BC}_{1}$ population.

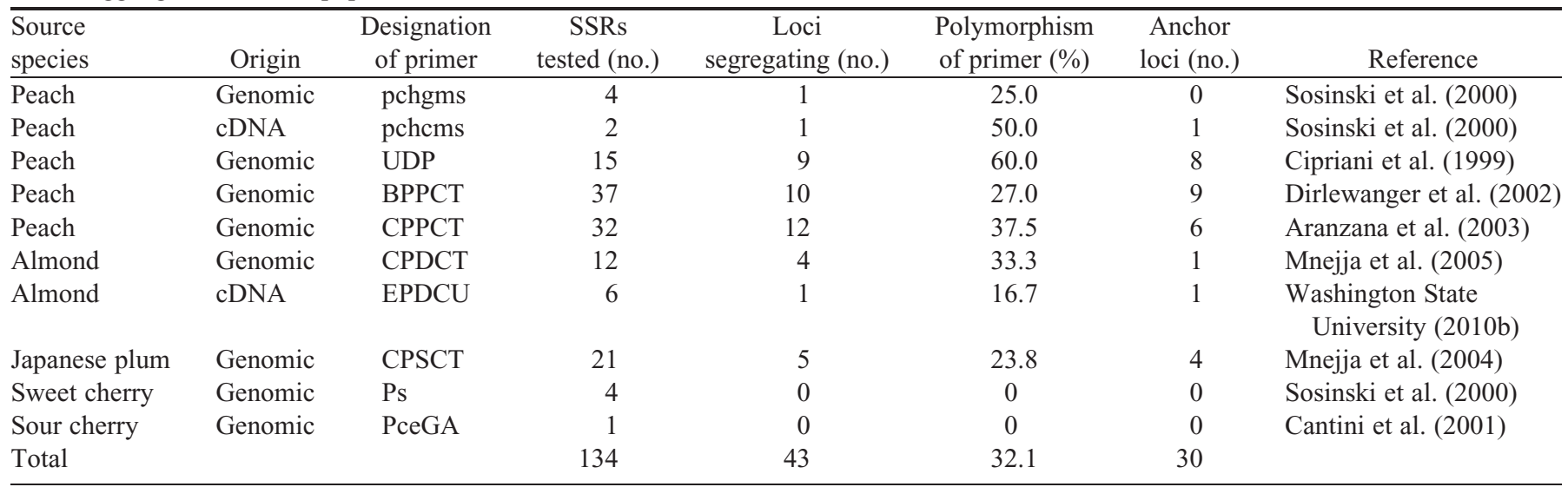


Table 3. The sequence information of resistance gene analog (RGA) specific primers used for polymerase chain reactions in the seedling of the Prunus kansuensis 'Honggengansutao' $\mathrm{BC}_{1}$ population.

\begin{tabular}{|c|c|c|c|}
\hline RGA & Forward primer $\left(5^{\prime}-3^{\prime}\right)$ & Reverse primer $\left(5^{\prime}-3^{\prime}\right)$ & $\begin{array}{c}\text { Amplification } \\
\text { size (bp) }\end{array}$ \\
\hline$\overline{\text { NBS1 }}$ & GAAGATTGAGGAGCGCTTTG & GGGAGGCCCTTACACTTCTC & 459 \\
\hline NBS3 & TTTTGACAGCATCCGTGCTA & TCCACTCCAAATGTGCTCAG & 320 \\
\hline NBS4 & GGTGTGGGAAAGACGACAAT & TAGCGTATTGCACGTTCTGC & 486 \\
\hline NBS5 & GGGGTTTCTCAACGTGGTAA & GGAGGCCTCTGGCATAATTT & 409 \\
\hline NBS9 & GGGGGAGTGGGAAAGACTAC & TTTCTTAAAGGCATGTCGCC & 420 \\
\hline NBS10 & CTGAGAATGCTTTGCTGCTG & ATTCCACGAAGCAAACAAGG & 310 \\
\hline NBS11 & AGGAGGGAAAGACTTGGAGC & GTCTTTCTCCTGGAAAGGCA & 293 \\
\hline NBS15 & GGAGCTCTTTAGTTGGCACGCT & GAGCCAGGGGAAGCCCTCCA & 104 \\
\hline NBS16 & AGGGATTTGGCGATGACGAGGC & CCTTGAGGGCGsAGTGGAAGGC & 135 \\
\hline NBS17 & AGCTGAGAATGCTTTGCCGC & TGGGTGGGCTTGGGAAAACGA & 435 \\
\hline NBS18 & CTTGAGCGCCAGGGGCAGTC & TGGAGCTCTTCAGTTGGCACGC & 110 \\
\hline NBS19 & AGGGCGAGTGGGAGACCCTT & CGGGAAGACCACCCTTGCGG & 504 \\
\hline NBS20 & AGCTCCCACGCATCACCCCT & CGTGTGGGATGTCCACCTTTGGG & 171 \\
\hline NBS21 & GGGATGGGTGGGCTGGGAAA & GCAAGTGGGAGGCCTTGAGCA & 494 \\
\hline NBS22 & TGGTTTGGTTCAGGCAGCAGA & CTAGGGGGAGCCCGTCAGCA & 220 \\
\hline NBS23 & GGTGCGATTGGTTTGGTTTGGGC & GCAAGGGGCAGACCTCCAGC & 230 \\
\hline NBS25 & GGGGGAGTGGGAAAGACTACACT & AGAGCGAGGGGGAGGCCTTT & 493 \\
\hline NBS26 & ACGGGTTCTCCTTGTTCTCGATG & CGAGGGGGAGTCCTCCGCAA & 287 \\
\hline NBS28 & GGGATGGGCGGATTGGGCAAA & CGAGGGGTAGGCCTCTGGCA & 510 \\
\hline NBS29 & GCGCTAGGGGAAGGCCATCAG & GGTGTGGACCAATTGGGGCAGTT & 262 \\
\hline NBS30 & GGGAAAGACTTGGAGCTGGCCC & AGGGCTAGAGGTAGGCCTCG & 360 \\
\hline NBS32 & GGGAGGGGTGGGTAAGACGACC & AGGTTCCCCGCTGTTCATCCCA & 194 \\
\hline
\end{tabular}

HQ263295-HQ263322). Their deduced amino acid sequences contained the characteristic motifs of NBS-type proteins such as P-loop, motif kinase-2, kinase-3, and GLPL motifs. Analysis using RGA-STS was performed on 22 RGAs selected randomly with gene-specific primers designed by the primer-blast program (Table 3).

Of the 22 RGA-STS primer pairs used in the PCR reactions, eight had complex profiles between 'Honggengansutao' and 'Bailey', seven have no amplification bands, two revealed no polymorphisms, whereas only five amplified DNA fragments that were polymorphic and used to map the $\mathrm{BC}_{1}$ population (Table 4). RGA-STS markers NBS1 and NBS16, which amplified bands but were not polymorphic, were converted to CAP markers using AluI and HaeIII, respectively.

LINKAGE MAP CONSTRUCTION. A total of 161 genetic markers (38 SSRs, 115 SRAPs, seven RGA-STSs, and one morphological marker for RKN resistance) was analyzed with chi-square tests of goodness of fit and used for the linkage map construction of 'Honggengansutao'. All but 23 loci were assigned to eight linkage groups. The linkage map comprised 138 loci (30 SSRs, 102 SRAPs, five RGA-STS, and PkMi gene) (Fig. 1). The map covered $616 \mathrm{cM}$ of the peach genome with an average marker spacing of $4.9 \mathrm{cM}$. The average number of loci per linkage group was 17.25 and ranged from $10(\mathrm{G} 5)$ to $24(\mathrm{G} 1$ or $\mathrm{G} 2)$. The length of each linkage group ranged from $65 \mathrm{cM}(\mathrm{G} 3$ or G8) to $104 \mathrm{cM}(\mathrm{G} 1)$. Linkage group G2 had the highest marker density with an average distance of $3.9 \mathrm{cM}$ per marker resulting from the presence of several cosegregating markers, whereas the least saturated linkage group G5 averaged $8.2 \mathrm{cM}$ per marker. Seven gaps (greater than $10 \mathrm{cM}$ ) ranging from 11 to $17 \mathrm{cM}$ were observed in this map (Fig. 1).

Comparative analysis with the PrUnUS Reference map. Thirty SSR markers were common to the Prunus reference map $\mathrm{T} \times \mathrm{E}$ ['Texas' almond $\times$ 'Earlygold' peach (Joobeur et al., 1998)] allowing for comparative analysis. SSR markers in G4 were not syntenic. The distance between other markers was calculated based on colinear loci. In the 'Honggengansutao' $x$ 'Bailey' $(\mathrm{H} \times \mathrm{B})$ map, $233 \mathrm{cM}(37.8 \%$ of the total length $)$ was anchored by common SSRs and in the $\mathrm{T} \times \mathrm{E}$ map, $213 \mathrm{cM}$ ( $40.7 \%$ of the total length). On average, $\mathrm{H} \times \mathrm{B}$ distances were 1.06-fold longer than the $\mathrm{T} \times \mathrm{E}$ distances. Among the linkage groups, this rate ranged from 0.49 for G6 to 2.58 for G1 (Table 5).

The location of the root-knot nematode $\boldsymbol{R}$ gene. The $P k M i$ gene controlling the nematode resistance from 'Honggengansutao' peach was mapped to linkage group G2 between NBS29 $(6 \mathrm{cM})$ and the SRAPs M4E11-100 (2.0 cM) (LOD 
G1

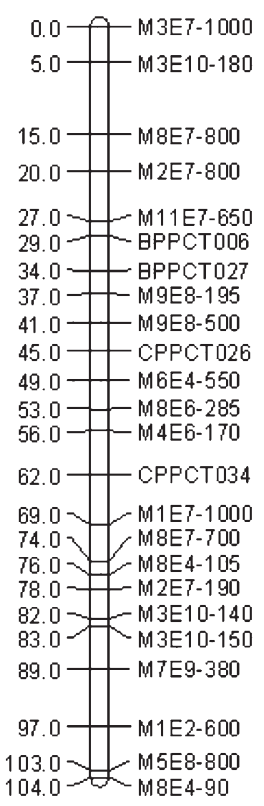

G2

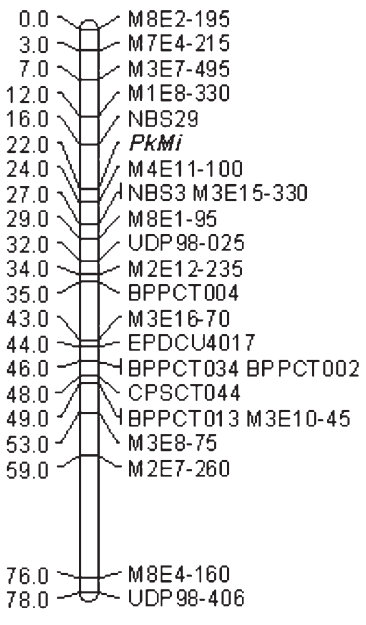

$78.0-1009$
G3

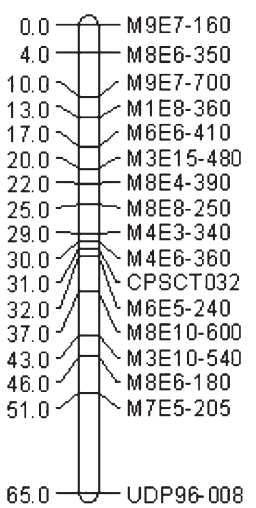

G6

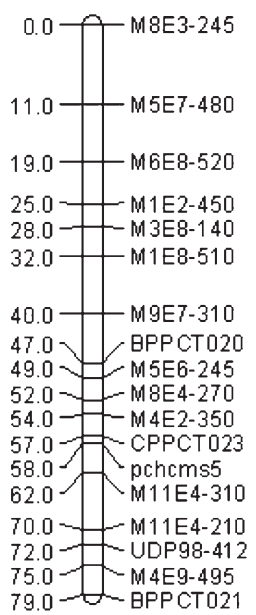

G4

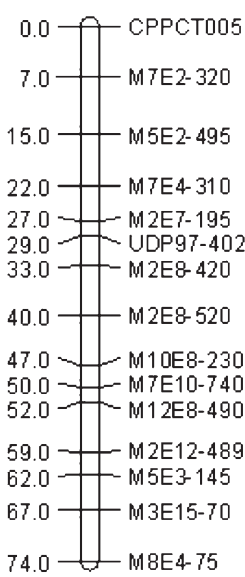

G7

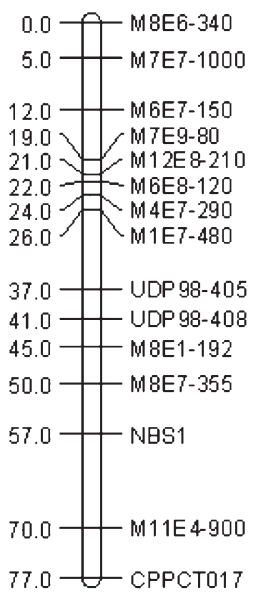

G5

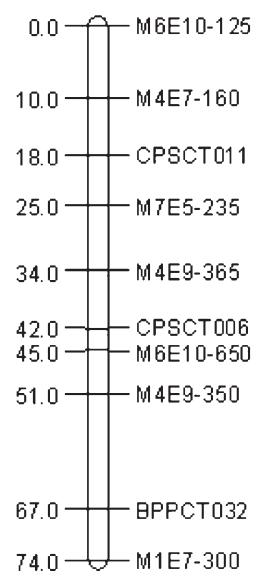

G8

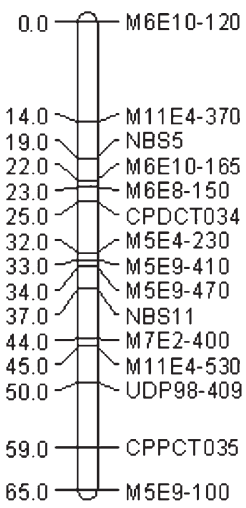

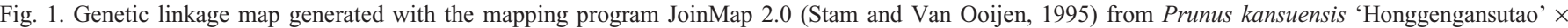
P. persica 'Bailey' $\mathrm{BC}_{1}$ population. Numbers identifying the linkage groups are shown on the top of each group. The names of the loci are shown on the right. Distance is shown on the left in centimorgans.

Table 5. Comparison distances covered by the common loci in every linkage group between Prunus kansuensis 'Honggengansutao' $\times P$. persica 'Bailey' $(\mathrm{H} \times \mathrm{B})$ and $P$. communis 'Texas' $\times P$. persica 'Earlygold' $(\mathrm{T} \times \mathrm{E})$ linkage maps.

\begin{tabular}{llcrrc}
\hline Group & \multicolumn{1}{c}{ Segments } & $\begin{array}{c}\mathrm{H} \times \mathrm{B} \\
\text { distances } \\
(\mathrm{cM})^{\mathrm{z}}\end{array}$ & $\begin{array}{c}\mathrm{T} \times \mathrm{E} \\
\text { distances } \\
(\mathrm{cM})^{\mathrm{y}}\end{array}$ & $\begin{array}{c}\text { Difference } \\
(\mathrm{cM})\end{array}$ & $\begin{array}{c}\text { Ratio } \\
\mathrm{H} \times \mathrm{B} / \mathrm{T} \times \mathrm{E}\end{array}$ \\
\hline G1 & CPPCT026-CPPCT034 & 17.0 & 6.6 & 10.4 & 2.58 \\
G2 & BPPCT004-UDP98-406 & 43.0 & 60.0 & -17.0 & 0.72 \\
G3 & CPSCT032-UDP96-008 & 34.0 & 17.5 & 16.5 & 1.94 \\
G5 & CPSCT011-BPPCT032 & 49.0 & 29.5 & 19.5 & 1.66 \\
G6 & CPPCT023-UDP98-412 & 15.0 & 30.5 & -15.5 & 0.49 \\
G7 & UDP98-405-CPPCT017 & 40.0 & 39.5 & 0.5 & 1.01 \\
G8 & CPDCT034-UDP98-409 & 25.0 & 27.7 & -2.7 & 0.90 \\
Total & & 223.0 & 211.3 & 11.7 & 1.06 \\
\hline
\end{tabular}

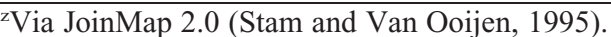

${ }^{\mathrm{y}}$ Via MapMaker 3.0 (Lander et al., 1987).

greater than 3.0). The nearest RGA-STS was NBS3 $(5 \mathrm{cM})$ and the nearest SSR marker was UDP98-025 (10 cM).

Physical distribution of all Resistance gene anAlogs in Chromosome of 'Honggengansutao' Peach. Five of seven
RGA-STS markers were mapped to G2, G7, and G8 (Fig. 1) through linkage analysis. BLASTN analysis of the five RGAs on the peach genome sequence was used to determine chromosome location. Location of the RGA-STS markers within the physical map by BLASTN analysis was consistent with genetic linkage mapping results (Table 6), indicating that BLASTN analysis on the peach genome sequence may be a shortcut for RGA mapping. BLASTN analysis of all RGA-STS markers against the 'Lovell' peach was used to determine the distribution of all RGAs on peach chromosomes. The results showed that highly similar homologs for 46 of 48 RGA sequences within the peach genome sequence were found (Table 7), whereas an acceptable E-value $\left(10^{-4}\right)$ homologs could not be found for only two RGA sequences (NBS62, NBS69). Different RGAs were sometimes 
Table 6. Comparison of the distribution of the five resistance gene analog (RGA)-sequence-tagged site (STS) markers in the Prunus kansuensis 'Honggengansutao' $\times P$. persica 'Bailey' $(\mathrm{H} \times \mathrm{B})$ map by linkage analysis and peach genome by blast analysis, respectively.

\begin{tabular}{|c|c|c|c|c|c|}
\hline RGAs & Length (bp) & $\begin{array}{l}\mathrm{H} \times \mathrm{B} \text { genetic } \\
\text { map location }\end{array}$ & Peach genome location & Blastn identities & $\begin{array}{c}\text { Blastn } \\
\text { E-value }\end{array}$ \\
\hline NBS1 & 528 & G7 (57.0 cM) & scaffold_7 (17074521-17074700 bp) & $480 / 482(99 \%)$ & 0 \\
\hline NBS3 & 516 & $\mathrm{G} 2(27.0 \mathrm{cM})$ & scaffold_2 (5656463-5656882 bp) & $457 / 467(97 \%)$ & 0 \\
\hline NBS11 & 528 & G8 $(37.0 \mathrm{cM})$ & scaffold_8 (13206393-13206890 bp) & $487 / 498(97 \%)$ & 0 \\
\hline NBS29 & 504 & G2 $(16.0 \mathrm{cM})$ & scaffold_2 (8908157-8908632 bp) & $466 / 476(97 \%)$ & 0 \\
\hline
\end{tabular}

Table 7. Physical distribution of 48 resistance gene analog (RGA) sequences in the peach genome.

\begin{tabular}{|c|c|c|c|}
\hline Location & RGA & RGAs and RGAs with the same loci & $\begin{array}{l}\text { RGAs with } \\
\text { different } \\
\text { loci (no.) }\end{array}$ \\
\hline$\overline{\text { Scaffold_1 }}$ & NBS40, NBS48 & & 2 \\
\hline Scaffold_2 & NBS3, NBS16, NBS21, NBS29, NBS38, NBS47 & NBS21 and NBS38; NBS29 and NBS47 & 4 \\
\hline Scaffold_3 & NBS72 & & 1 \\
\hline Scaffold_4 & NBS18 & & 1 \\
\hline Scaffold_5 & NBS4, NBS57, NBS64 & NBS4, NBS57 and NBS64 & 1 \\
\hline Scaffold_6 & NBS20, NBS37, NBS43, NBS51, NBS63, NBS67 & NBS37, NBS43, NBS51, and NBS67 & 3 \\
\hline Scaffold_7 & NBS1, NBS15, NBS26 & & 3 \\
\hline Scaffold_8 & $\begin{array}{l}\text { NBS5, NBS9, NBS10, NBS11, NBS17, NBS22, } \\
\text { NBS23, NBS25, NBS28, NBS30, NBS32, NBS36, } \\
\text { NBS39, NBS45, NBS49, NBS50, NBS53, NBS56, } \\
\text { NBS60, NBS61, NBS68 }\end{array}$ & $\begin{array}{l}\text { NBS9 and NBS25; NBS28 and NBS32; } \\
\text { NBS11 and NBS30; NBS39, NBS45, NBS49, } \\
\text { NBS50 and NBS61; NBS60 and NBS68 }\end{array}$ & 13 \\
\hline Scaffold_10 & NBS41 & & 1 \\
\hline Scaffold_29 & NBS19, NBS34 & NBS19 and NBS34 & 1 \\
\hline No scaffold & NBS62, NBS69 & & \\
\hline Total & & & 30 \\
\hline
\end{tabular}

most similar to the same locus within the RGA analysis. This could also occur as a result of PCR artifacts or if genes are duplicated in 'Honggengansutao' versus the peach reference genome. After filtering the "false" RGAs, 28 RGAs were placed on scaffold_1 to scaffold_8. Scaffold_8 had the highest RGA density, and Scaffolds_3, Scaffolds_4, and Scaffolds_5 had the lowest.

\section{Discussion}

Several intraspecific and interspecific Prunus maps, developed using different molecular markers (Dirlewanger et al., 1998, 2004; Jauregui et al., 2001; Lu et al., 1998), provide a framework for construction of a highly saturated molecular genetic map. The most recent linkage map was constructed by a controlled $\mathrm{F}_{2}$ cross between 'Guardian' and 'Nemaguard' (Blenda et al., 2007). This map covers a genetic distance of $737 \mathrm{cM}$ with an average marker spacing of $4.7 \mathrm{cM}$. In the present study, the $\mathrm{H} \times \mathrm{B}$ map covers $616 \mathrm{cM}$ of the peach genome with an average marker spacing of $4.9 \mathrm{cM}$. The genetic distances were up to 1.19-fold longer in the 'Guardian' $\times$ 'Nemaguard' map than in the $\mathrm{H} \times \mathrm{B}$ map, possibly as a result of a decreased chiasmata frequency resulting from reduced homology between the DNA strands (Borts and Haber, 1987).

Considering all the 134 SSRs tested in our study, we observed a polymorphism rate of $32.1 \%$, which is a value significantly lower than that reported in other Prunus progenies (Aranzana et al., 2003; Lambert et al., 2004). This rate increased to $36.7 \%$ (33 of 90) for peach primer pairs (pchgms, pchcms, UDP, BPPCT, and CPPCT). These results demonstrated that SSRs derived from almond, plum, and cherry had a low polymorphism in peach.

Loci rearrangement was observed in the $\mathrm{H} \times \mathrm{B}$ map. Two SSR markers, CPPCT005 and UDP97-402, were mapped within the same linkage group (G4) in the present study. However, the CPPCT005 was mapped in G2 for the $\mathrm{J} \times \mathrm{F}$ map and in G4 for the $\mathrm{T} \times \mathrm{E}$ map, whereas UDP97-402 was positioned in G4 for the $\mathrm{J} \times \mathrm{F}$ map and $\mathrm{G} 2$ for the $\mathrm{T} \times \mathrm{E}$ map. The possibility maybe is that there are two or more alleles of these primers, for example UDP97-402a (G4) and UDP97-402b (G2) in the $\mathrm{R} \times \mathrm{D}$ linkage map (Sanchez-Perez et al., 2005). The rearrangement of CPPCT005 in different linkage groups could aid to a more precise definition of the location of it.

Limited segregation was observed for SSR markers, so the SRAP technique was used to increase the density of markers in our study. These SRAP markers have been shown to be reproducible, reliable, and locus-specific markers for rapid increase of the coverage of genetic maps (Li and Quiros, 2001). By adding SRAPs to the map developed by SSR markers, we were able to generate maps that are comparatively longer than the $\mathrm{T} \times$ E map (519 cM) generated with SSRs and RFLPs alone (Dirlewanger et al., 2004). This increase in the total length was mainly caused by the addition of markers to regions near the edge of some groups where SSRs were poorly represented, including the top regions on G1, G2, G3, G6, G7, and G8 and the bottom of $\mathrm{G} 1$ and $\mathrm{G} 4$ in the $\mathrm{H} \times \mathrm{B}$ map.

A single gene, designated RMia, controlling resistance to both MA and MI, is mapped on linkage Group 2 of the Prunus 
reference map and $\approx 10 \mathrm{cM}$ with UDP98-025 (Dirlewanger et al., 2004). In our study, an $R$ gene to MA, $P k M i$, was mapped onto the same region of Group 2 by using an intraspecific $\mathrm{BC}_{1}$ population cross between $P$. persica and $P$. kansuensis, and for the nearest SSR marker, UDP98-025, the distance is also $10 \mathrm{cM}$. Our results suggest that the $R$ gene in 'Honggengansutao' is an allele of $R M i a$. This is the first report of an $R$ gene for RKN in P. kansuensis.

Evaluation of RKN resistance is a time- and labor-intensive process. Therefore, molecular markers tightly linked to the nematode $R$ genes are of special interest for breeding and improving peach rootstocks. Several SSR markers around the loci of the $R$ gene were screened in the $\mathrm{BC}_{1}$ population but most showed no polymorphism. The nearest SSR marker, UDP98-025 at $10 \mathrm{cM}$, had a greater genetic distance to resistance loci, and it may be of little use. Additional markers saturating the region around $P k M i$ are necessary for marker-assisted selection (MAS).

Many sources of nematode resistance have been identified and major resistance loci have been described and mapped in diverse plant crops. These $R$ genes include $M i-1$ (Milligan et al., 1998) and Hero (Karin et al., 2002) in tomato and Cre3 (Lagudah et al., 1997) in wheat (Triticum aestivum). Map-based cloning has also defined an $R$ gene in Prunus species. The other study proved that the RKN $R$ gene $(M a)$ in Myrobalan plum (another resistance resource in Prunus) belongs to a cluster of NBS-LRR candidate genes (Claverie et al., 2006; Esmenjaud and Dirlewanger, 2007); however, Ma has not been sequenced. What about Rmia? The report in our study may be helpful to analyze the sequence of the Rmia gene.

In this article, we describe the homologous sequence cloning of partial NBS sequences from RGA using degenerate primers and anchored all cloned RGAs on peach genetic linkage map and genome for creating a $R$ gene map to map the $P k M i$ gene. In a previously constructed resistance map (Lalli et al., 2005), a total of 42 regions of resistance mapped onto seven of the eight linkage groups (RGAs were absent in Group 3) and the RGA in the Rmia region of Group 2 belonged to a non-TIR type. In our study, a resistance genetic map for peach was generated through linkage analysis and currently contains five RGAs distributed to G2, G7, and G8 (Fig. 1). Twenty-eight RGAs, which placed on scaffold_1 to scaffold_8 by BLAST analysis with a peach genome sequence (Table 7), identified putative RGAs within the genome. The RGAs NBS3 and NBS29, mapped in the region of the RKN $R$ gene, belong to the TIR type characterized by the presence of aspartate residues (D) instead of tryptophan residues (W) in the final position of kinase-2. The development of a resistance map for Prunus provides resistance loci for designing breeding strategies to yield broadly resistant crops (Lalli et al., 2005).

\section{Literature Cited}

Aarts, M.G.M., B.L. Hekkert, E.B. Holub, J.L. Beynon, W.J. Stiekema, and A. Pereira. 1998. Identification of R-gene homologous DNA fragments genetically linked to disease resistance loci in Arabidopsis thaliana. Mol. Plant Microbe Interact. 11:251-258.

Aranzana, M.J., A. Pineda, P. Cosson, E. Dirlewanger, J. Ascasibar, G. Cipriani, C.D. Ryder, R. Testolin, A.G. Abbott, G.J. King, A.F. Iezzoni, and P. Arus. 2003. A set of simple-sequence repeat (SSR) markers covering the Prunus genome. Theor. Appl. Genet. 87:805-815.

Barker, K.R. 1985. Design of greenhouse and microplot experiments for evaluation of plant resistance to nematodes, p. 103-113. In: Zuckerman, B.M., W.F. Mai, and M.B. Harrison (eds.). Plant nematology laboratory manual. Univ. of Massachusetts Agr. Expt. Sta., Amherst, MA.

Blenda, A.V., I. Verde, L.L. Georgi, G.L. Reighard, S.D. Forrest, M. Munoz-Torres, W.V. Baird, and A.G. Abbott. 2007. Construction of a genetic linkage map and identification of molecular markers in peach rootstocks for response to peach tree short life syndrome. Tree Genet. Genomes 3:341-350.

Borts, R.H. and J.E. Haber. 1987. Meiotic recombination in yeast: Alteration by multiple heterozygosities. Science 237:1459-1465.

Cantini, C., A.F. Iezzoni, W.F. Lamboy, M. Boritzki, and D. Struss. 2001. DNA fingerprinting of tetraploid cherry germplasm using simple sequence repeats. J. Amer. Soc. Hort. Sci. 126:205-209.

Cipriani, G., G. Lot, W.G. Huang, M.T. Marrazzo, E. Peterlunger, and R. Testolin. 1999. AC/GT and AG/CT microsatellite repeats in peach [Prunus persica (L.) Batsch]: Isolation, characterisation and crossspecies amplification in Prunus. Theor. Appl. Genet. 99:65-72.

Claverie, M., N. Bosselut, A.C. Lecouls, R. Voisin, C. Poizat, E. Dirlewanger, M. Kleinhentz, B. Lafargue, F. Laigret, and D. Esmenjaud. 2004. Location of independent root-knot nematode resistance genes in plum and peach. Theor. Appl. Genet. 108:765-773.

Claverie, M., E. Dirlewanger, P. Cosson, N. Bosselut, A.C. Lecouls, R. Voisin, M. Kleinhentz, B. Lafargue, M.N. Rosso, P. Abad, C. Chalhoub, and D. Esmenjaud. 2006. The Ma gene from Myrobalan plum (Prunus cerasifera Ehr.) conferring a complete-spectrum resistance to rootknot nematodes (Meloidogyne spp.) is a member of a TIR-NBS LRR gene cluster, 28th Symp. European Soc. Nematol., Blagoevgrad, Bulgaria, 5-9 June 2006.

Dangl, J.L. and J.D. Jones. 2001. Plant pathogens and integrated defence responses to infection. Nature 411:826-833.

Desmond, R.L. and B. Daniele. 2008. The peach: Botany, production and uses. CAB International, London, UK.

Dirlewanger, E., P. Cosson, M. Tavaud, M.J. Aranzana, C. Poizat, A. Zanetto, P. Arus, and F. Laigret. 2002. Development of microsatellite markers in peach [Prunus persica (L.) Batsch] and their use in genetic diversity analysis in peach and sweet cherry (Prunus avium L.). Theor. Appl. Genet. 105:127-138.

Dirlewanger, E., E. Graziano, T. Joobeur, F. Garriga-Caldere, P. Cosson, W. Howad, and P. Arus. 2004. Comparative mapping and marker assisted selection in Rosaceae fruit crops. Proc. Natl. Acad. Sci. USA 101:9891-9896.

Dirlewanger, E., V. Pronier, C. Parvery, C. Rothan, A. Guye, and R. Monet. 1998. Genetic linkage map of peach [Prunus persica (L.) Batsch] using morphological and molecular markers. Theor. Appl. Genet. 97:888-895.

Esmenjaud, D. and E. Dirlewanger. 2007. Plum, p. 119-135. In: Kole, C. (ed.). Genome mapping and molecular breeding in plants, Vol. 4 (Fruits and nuts). Springer, Heidelberg, Germany.

Esmenjaud, D., J.C. Minot, R. Voisin, J. Pinochet, M.H. Simard, and G. Salesses. 1997. Differential response to root-knot nematodes in Prunus species and correlative genetic implications. J. Nematol. 29:370-380. Gaspero, G.D., G. Cipriani, A.F. Adam-Blondon, and R. Testolin. 2007. Linkage maps of grapevine displaying the chromosomal locations of 420 microsatellite markers and 82 markers for R-gene candidates. Theor. Appl. Genet. 114:1249-1263.

Hinchliffe, D.J., Y.Z. Lu, C. Potenza, C. Segupta-Gopalan, R.G. Cantrell, and J.F. Zhang. 2005. Resistance gene analogue markers are mapped to homeologous chromosomes in cultivated tetraploid cotton. Theor. Appl. Genet. 110:1074-1085.

Jauregui, B., M.C. Vicente, R. Messeguer, A. Felipe, A. Bonnet, G. Salesses, and P. Arus. 2001. A reciprocal translocation between 'Garfi' almond and 'Nemared' peach. Theor. Appl. Genet. 102:1169-1176.

Joobeur, T., M.A. Viruel, M.C. De Vicente, B. Jauregui, J. Ballester, M.T. Dettori, I. Verde, M.J. Troco, R. Messeguer, I. Battle, R. Quarta, E. Dirlewanger, and P. Arus. 1998. Construction of a saturated linkage map for Prunus using an almond $\times$ peach $\mathrm{F}_{2}$ progeny. Theor. Appl. Genet. 97:1034-1041.

Karin, E., K. Amar, K. Doris, K. Dorothee, S.P. Mark, and W.G. Martin. 2002. The broad-spectrum potato cyst nematode resistance 
gene (Hero) from tomato is the only member of a large gene family of NBS-LRR genes with an unusual amino acid repeat in the LRR region. Plant J. 31:127-136.

Lagudah, E.S., O. Moullet, and R. Appels. 1997. Map-based cloning of a gene sequence encoding a nucleotide-binding domain and a leucine-rich region at the $\mathrm{Cre} 3$ nematode resistance locus of wheat. Genome 40:659-665.

Lalli, D.A., V. Decroocq, A.V. Blenda, V. Schurdi-Levraud, L. Garay, O. Le Gall, V. Damsteegt, G.L. Reighard, and A.G. Abbott. 2005. Identification and mapping of resistance gene analogs (RGAs) in Prunus: A resistance map for Prunus. Theor. Appl. Genet. 111:15041513.

Lambert, P., L.S. Hagen, P. Arus, and J.M. Audergon. 2004. Genetic linkage maps of two apricot cultivars (Prunus armeniaca L.) compared with the almond Texas $\times$ peach Earlygold reference map for Prunus. Theor. Appl. Genet. 108:1120-1130.

Lamberti, F. 1979. Economic importance of Meloidogyne spp. in subtropical and Mediterranean climates, p. 342-357. In: Lamberti, F. and C.E. Taylor (eds.). Root-knot nematodes (Meloidogyne spp.): Systematics, biology and control. Academic Press, New York, NY. Lander, E., P. Green, J. Abrahamson, A. Barlow, M. Daley, S. Lincoln, and L. Newburg. 1987. MAPMAKER: An interactive computer package for constructing primary genetic linkage maps of experimental and natural populations. Genomics 1:174-181.

Lecouls, A.C., G. Salesses, J.C. Minot, R. Voisin, A. Bonnet, and D. Esmenjaud. 1997. Spectrum of the Ma genes for resistance to Meloidogyne spp. in Myrobalan plum. Theor. Appl. Genet. 85:1325-1334.

Li, G. and C.F. Quiros. 2001. Sequence-related amplified polymorphism (SRAP), a new marker system based on a simple PCR reaction: Its application to mapping and gene tagging in Brassica. Theor. Appl. Genet. 103:455-461.

Lu, Z.X., B. Sosinski, G.L. Reighard, W.V. Baird, and A.G. Abbott. 1998. Construction of a genetic linkage map and identification of AFLP markers for resistance to root-knot nematodes in peach rootstock. Genome 41:199-207.

Milligan, S., J. Bodeau, J. Yaghoobi, I. Kaloshian, P. Zabel, and V.M. Williamson. 1998. The root-knot nematode resistance gene $M i$ from tomato is a member of the leucine zipper, nucleotide binding, leucine-rich repeat family of plant genes. Plant Cell 10:1307-1319.

Mnejja, M., J. Garcia-Mas, W. Howad, and P. Arus. 2005. Development and transportability across Prunus species of 42 polymorphic almond microsatellites. Mol. Ecol. Notes 5:531-535.

Mnejja, M., J. Garcia-Mas, W. Howad, M.L. Badenes, and P. Arus. 2004. Simple sequence repeat (SSR) marker of japanese plum (Prunus salicina Lindl.) are highly polymorphic and transferable to peach and almond. Mol. Ecol. Notes 4:163-166.

National Center for Biotechnology Information. 2010a. Screen a sequence using Vecscreen. 10 June 2010. <http://www.ncbi.nlm.nih. gov/VecScreen/>.
National Center for Biotechnology Information. 2010b. Primer-blast: A tool for finding specific primers. 10 June 2010. $<$ http://www.ncbi. nlm.nih.gov/tools/primer-blast/>.

Qiang, X., X.P. Wen, and X.X. Deng. 2005. Isolation of TIR and nonTIR NBS-LRR resistance gene analogues and identification of molecular markers linked to a powdery mildew resistance locus in chestnut rose (Rosa roxburghii Tratt). Theor. Appl. Genet. 111:819830.

Ramming, D.W. and O. Tanner. 1983. Nemared peach rootstock. HortScience 18:376.

Robert, N.G.M., J.B. David, C.B. Franc, M.S. Candice, C.A. Paulo, F.M. Natalia, C.T. Roberto, T.S. Manoel, and J.P. Georgios. 2008. Analysis of non-TIR NBS-LRR resistance gene analogs in Musa acuminata Colla: Isolation, RFLP marker development, and physical mapping. BMC Plant Biol. 8:15.

Rubio-Cabetas, M.J., J.C. Minot, R. Voisin, D. Esmenjaud, G. Salesses, and A. Bonnet. 1999. Response of the Ma genes from Myrobalan plum to Meloidogyne hapla and M. mayaguensis. HortScience 34:1266-1268.

Sanchez-Perez, R., D. Ruiz, F. Dicenta, J. Egea, and P. MartinezGomez. 2005. Application of simple sequence repeat (SSR) markers in apricot breeding: Molecular characterization, protection, and genetic relationships. Sci. Hort. 103:305-315.

Sasser, J.N. 1977. Worldwide dissemination and importance of the root-knot nematodes Meloidogyne spp. J. Nematol. 9:585-589.

Sosinski, B., M. Gannavarapu, L.D. Hager, L.E. Beck, G.J. King, C.D. Ryder, S. Rajapakse, W.V. Baird, R.E. Ballard, and A.G. Abbott. 2000. Characterization of microsatellite markers in peach [Prunus persica (L.) Batsch]. Theor. Appl. Genet. 101:421428.

Stam, P. and J.W. Van Ooijen. 1995. JoinMap ${ }^{\mathrm{TM}}$ version 2.0: Software for the calculation of genetic linkage maps. Dienst Landbouwkundig Onderzoek, Centre for Plant Breeding and Reproduction Research, Wageningen, The Netherlands.

Van Ghelder, C., B. Lafargue, E. Dirlewanger, A. Ouassa, R. Voisin, J. Polidori, M. Kleinhentz, and D. Esmenjaud. 2010. Characterization of the RMja gene for resistance to the root-knot nematodes in almond: Spectrum, location and interest for Prunus breeding. Tree Genet. Genomes 6:503-511.

Wang, Y., B. Rosen, J. Scoffield, M. Egnin, D. Mortley, S. Steiner, D.R. Cook, and G. He. 2010. Isolation and analysis of resistance gene homologues in sweetpotato. Plant Breed. 129:519-525.

Washington State University. 2010a. Blast you sequences. 10 June 2010. <http://www.rosaceae.org/bio/content?title=\&url=http://app. bioinfo.wsu.edu/blast/blast_noheader.html\&style=width:950px;height: 950px>.

Washington State University. 2010b. Marker search. 10 June 2010. $<$ http://www.rosaceae.org/bio/content/?title=\&url=/cgi-bin/gdr/ gdr_marker_search.cgi\&style=width:950px; height:1024px>. 